\title{
Schlesien in der Oberlausitz? Versuch einer Ortsbestimmung
}

\author{
Robert Lorenz
}

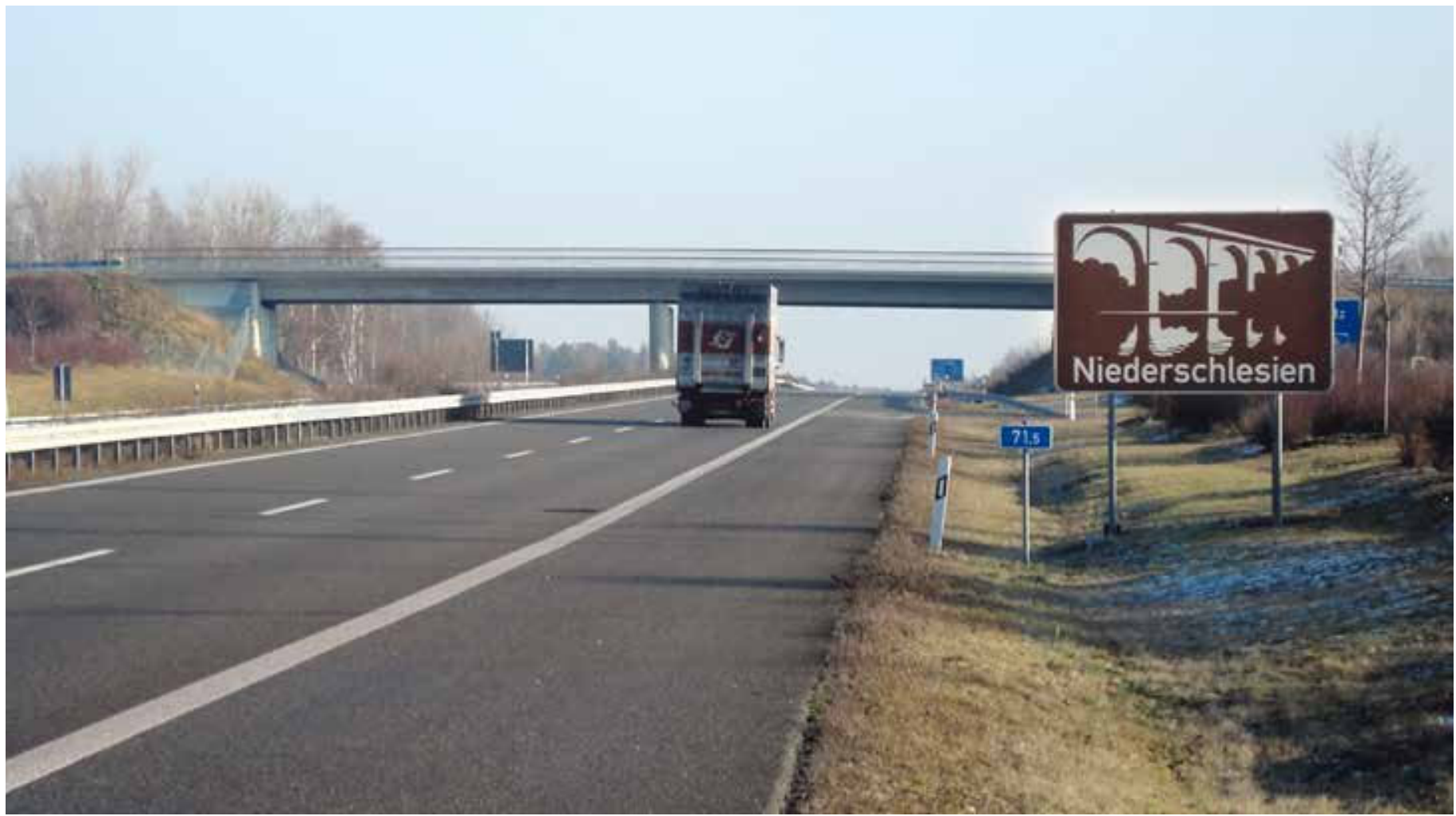

Beschilderung an der A4 Richtung Görlitz, 2014. Foto: Robert Lorenz
Die Oberlausitz und (Nieder-)Schlesien - es ist kaum möglich, in regionalhistorisch interessierten Kreisen unserer Region schneller eine hitzige Debatte vom Zaun zu brechen als durch die Nennung dieses Begriffspaares. Seit 1990 betonen viele Menschen vor allem im Städtedreieck Görlitz - Rothenburg Weißwasser ihre Identität als Schlesier und reklamieren zugleich ihren Teil der Oberlausitz als (nieder-)schlesisch. Seit 1992 sichert ihnen auch die Landesverfassung des Freistaates Sachsen dieses Recht zu - der größte Erfolg der schlesischen Identitätsbewegung an der Neiße nach 1990. Gleichwohl sind die Meinungsverschiedenheiten darüber, ob die Oberlausitz und Schlesien überhaupt in ein und demselben Raum „stattfinden“ können, nie verstummt. So fand bereits 1993 auf dem in Görlitz abgehaltenen zweiten „Tag der
Sachsen“ eine Podiumsdiskussion zu dieser Frage statt, die die Gemüter erhitzte. Offenbar ist Identität auch in diesem Fall einmal mehr Ansichts- und Gefühlssache, über die sich bekanntlich nur schwer mit rationaler Kühle diskutieren lässt.

Die Diskussion um den „schlesischen Charakter" der östlichen Oberlausitz ist bis heute nicht verstummt. Eine Erklärung hierfür liegt in einer zweifachen Herausforderung. Die erste ist eine regionale oberlausitzische. Denn es waren vor allem die Görlitzer, die auf der Suche nach einem Alleinstellungsmerkmal nach 1990 die „schlesischen Jahre“ ihrer Geschichte zwischen 1815 und 1945 betonten und diese gegenüber den Nachbarstädten Zittau und Bautzen sowie besonders stark gegenüber der Landeshauptstadt Dresden in einem sich pointiert „antisächsisch“ 
gebenden Tonfall hervorhoben. Damit folgte man in Görlitz einem Impuls, der sich durch die gesamte Stadtgeschichte hinweg beobachten lässt: dem Konkurrenzkampf innerhalb des Oberlausitzer Sechsstädtebundes um die Spitzenposition sowie dem Bedürfnis, sich gegenüber dem stets außerhalb der Oberlausitz gelegenen Regierungssitz zu behaupten - sei dieser nun Prag, Breslau oder eben Dresden. Die damit einhergehende Betonung des Schlesischen nahm dabei für die Oberlausitzer Nachbarn mitunter irritierende Züge an. Verlässt man beispielsweise seit 2004 auf der A4 an der westlichen Einfahrt in den Autobahntunnel der Königshainer Berge plötzlich die Oberlausitz, wie es der Name „Schlesisches Tor“ zu suggerieren scheint? Und befindet man sich als Spieler des FC Oberlausitz Neugersdorf auf dem Stadion "Junge Welt" des Niederschlesischen Fußballvereins Gelb-Weiß 09 Görlitz im „Fußballausland“, wenn man durch den Görlitzer Stadionsprecher als „Gast aus der Oberlausitz" begrüßt wird? Dass hierzu bis in die Gegenwart Diskussionsbedarf besteht, verdeutlichten nicht zuletzt die Geburtswehen des Landkreises Görlitz. 2008 zeichneten sich am Inhalt des viel diskutierten neuen Kreiswappens unterschiedliche Zugehörigkeitsgefühle im neuen Großkreis ab, obwohl doch alle Beteiligten der Landkarte nach in der Oberlausitz leben.

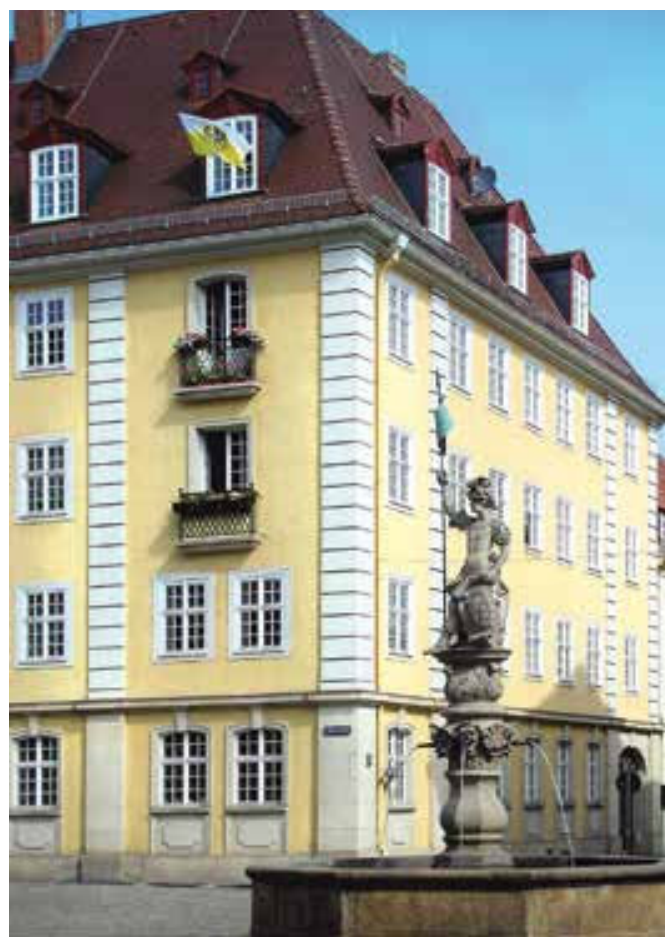

Schlesische Fahne am Obermarkt in Görlitz, 2011. Foto: Robert Lorenz

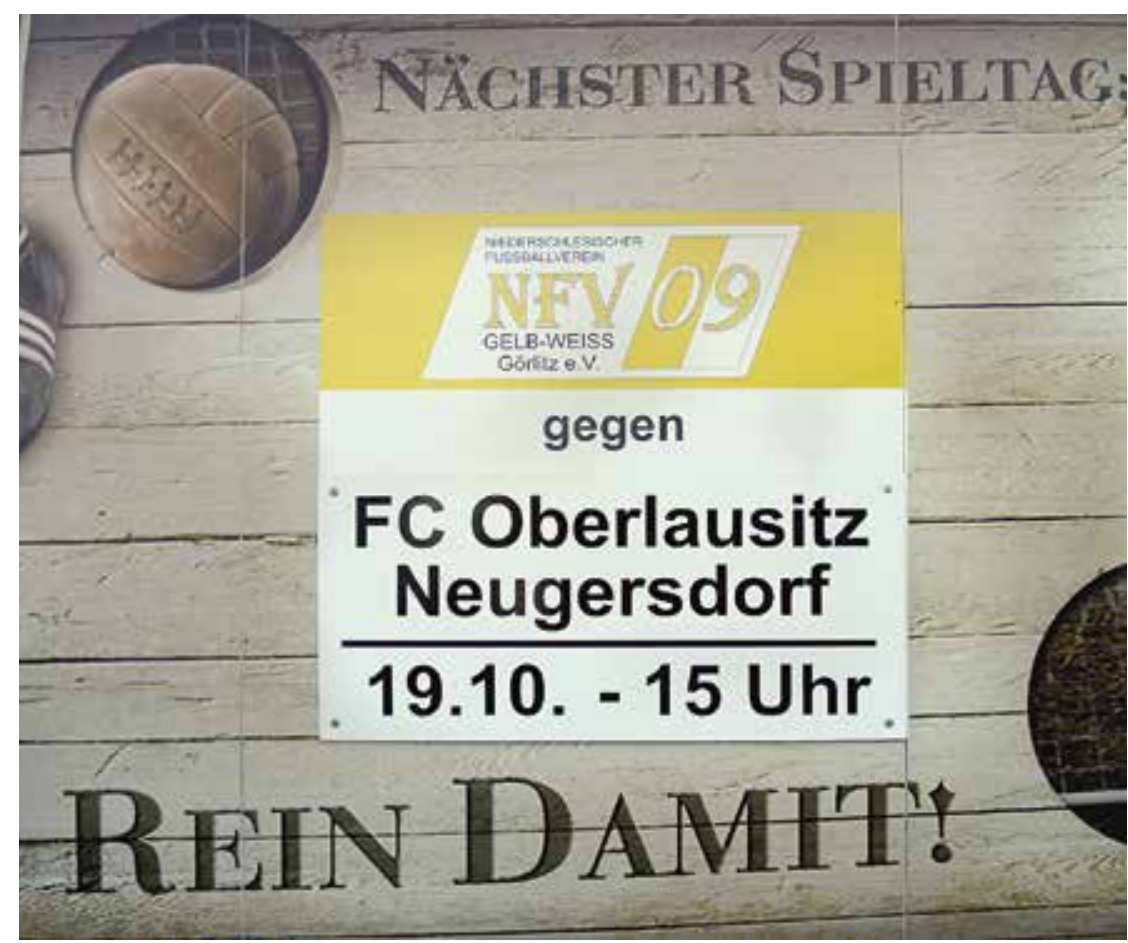

Die zweite Herausforderung weist über den regionalen Rahmen hinaus auf die Frage, wie sich das wiedervereinigte Deutschland zum Erbe seiner seit 1945 zur Republik Polen gehörenden ehemaligen östlichen Landesteile positioniert. Eine ganze Region am östlichen Rand Deutschlands definiert sich plötzlich offiziell als schlesisch. Dies beschwört bis auf den heutigen Tag Fragezeichen herauf, in denen sich zwei unterschiedliche, mit je eigenen Tabuisierungen und Blickverengungen versehene deutsche Verarbeitungs- wie Verdrängungstraditionen des Bruches von 1945 verdeutlichen. "Schlesien“ ist also in der Tat in der Gegenwart ein Mythos - sowohl in der Oberlausitz wie weit über sie hinaus.

\section{Der schlesische Nachbar}

Es fällt aus heutiger Perspektive schwer, sich in eine Zeit zurückzuversetzen, in der Schlesien ein selbstverständlicher Bestandteil der mentalen Landkarte der Deutschen war, in der Breslau als deutsche Großstadt galt und in der Gerhard Hauptmann auf seinen Bahnfahrten von Berlin ins heimische Riesengebirge hin und wieder einen Tag Pause in Görlitz einlegte, um im Haus der befreundeten jüdischen Familie Alexander-Katz zu übernachten, wie es Paul Mühsam in seinen Lebenserinnerungen beschreibt. Und auch die tägliche Bahnfahrt des jugendlichen Arno Schmidt vom östlichen Ende der Oberlausitz in Lauban zum Gymnasium nach Görlitz
Lokalderby der Fußballvereine auf der "Jungen Welt" in Görlitz, 2008. Foto: Robert Lorenz 
„Volksfreund aus der Oberlausitz" aus Niesky, 1893. Abbildung: Archiv Robert Lorenz

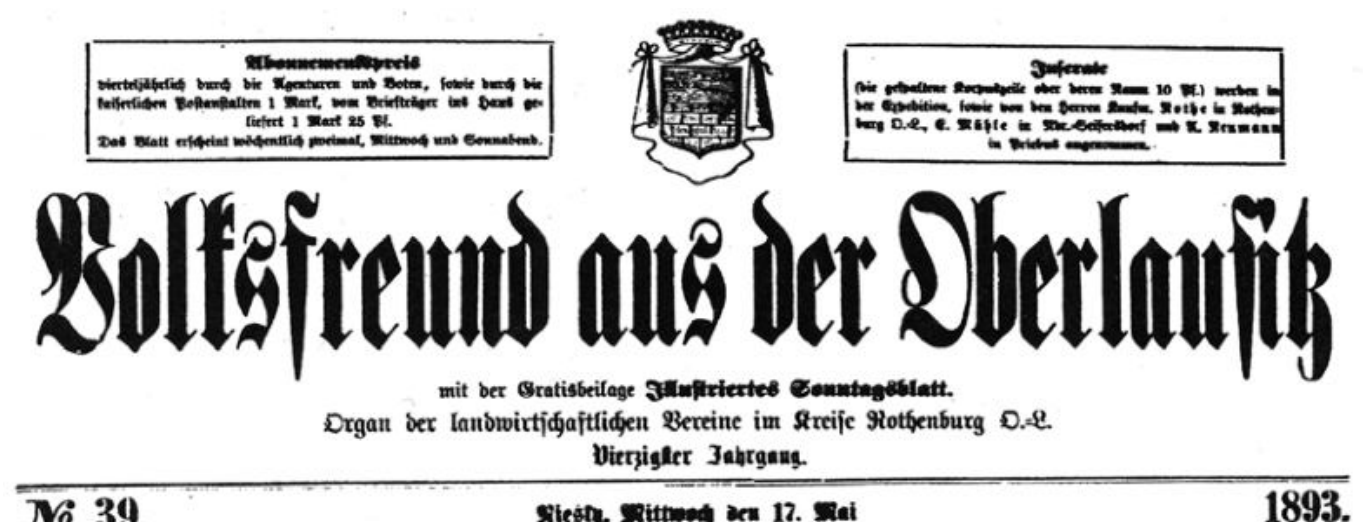

scheint in einer lange vergangenen und verschütteten Welt zu liegen. Diese Vorstellung von der einstigen Alltäglichkeit dieser räumlichen Bezüge gilt es aber festzuhalten, wenn man die Frage danach stellt, welche Rolle Schlesien für die Oberlausitz in der Zeit ihrer historisch gewachsenen Nachbarschaft spielte.

Zunächst muss daran erinnert werden, dass die Oberlausitz und Schlesien zwei unterschiedliche feudale Einheiten waren. Die Grenze zwischen beiden verlief über Jahrhunderte östlich von Lauban entlang des Queis. Der schlesische Adler und die goldene Mauer der Oberlausitz standen für voneinander unabhängige Herrschaftsgebiete. Das war auch in den Jahrhunderten der gemeinsamen Zugehörigkeit zur böhmischen Krone nicht anders - und so stehen die Wappen Schlesiens und der Oberlausitz am Hradschin und am Altstädter Brückenturm in Prag für zwei voneinander unabhängige Nebenländer Böhmens. Aus diesem Grund konnte Sachsen im Prager Frieden 1635 die Lausitzen von den Habsburgern erwerben, während Schlesien unter böhmischer Hoheit verblieb. Aus diesem Grund unterlag Schlesien nach dem Dreißigjährigen Krieg einer drakonischen Gegenreformation - und die Oberlausitz nicht. Und selbst 1844 besuchte der preußische König Friedrich Wilhelm IV. (17951861) die Stadt Görlitz noch als Markgraf der beiden Lausitzen. Erst im Dritten Reich wurden 1941 mit der Schaffung des Gaues Niederschlesien die letzten Reste der eigenständigen Ständeverwaltung im preußischen Teil der Oberlausitz endgültig abgeschafft.

Auch beim Blick auf die jeweilige Führungselite offenbaren sich klare Unterschiede zwischen beiden Ländern. Ohne die Bedeutung einiger Geschlechter der Oberlausitz schmälern zu wollen - machtpolitisch bewegte sich der schlesische Adel im Mittelalter auf einem anderen Niveau. Zu keinem Zeitpunkt ihrer
Geschichte besaß die Oberlausitz ein mit den schlesischen Piasten vergleichbares Fürstengeschlecht. Auch einen eigenen Bischof hatte das Land der Sechsstädte anders als Breslau nie aufzuweisen (und kirchlich gehörte man bis zur Reformation zu Meißen). Aus diesem Grund gelangte das städtische Bürgertum zwischen Pulsnitz und Queis zu außergewöhnlicher Kraft. Waren es in Schlesien die einzelnen Fürsten, die sich einen Kampf um Macht und Einfluss lieferten, so taten dies in den Sechsstädten der Oberlausitz die Ratsherren.

Es gibt also genügend Gründe, auf die strukturellen Unterschiedlichkeiten Schlesiens und der Oberlausitz hinzuweisen. Trotzdem wäre es übertrieben, aus ihnen einen unüberbrückbaren kulturellen Gegensatz zu konstruieren. Denn selbstverständlich standen beide Länder als unmittelbare Nachbarn in regem wirtschaftlichem wie gesellschaftlich-kulturellem Austausch. Landschaftlich ist der Übergang zwischen ihnen fließend, was auch für die Dialektlandschaft galt: das Ostlausitzische wurde bis weit hinauf nach Hirschberg gesprochen, sodass beispielsweise die Dialektsprache in Hauptmanns "Die Weber" vom Görlitzer Theaterpublikum gut verstanden werden konnte. Und letztlich teilte man als Nebenländer Böhmens die gleichen Vor- und Nachteile der aus Prager Perspektive abgeschiedenen Lage "hinter den Bergen“. Gemeinsam sah man sich den Überfällen der Hussiten ausgesetzt und erduldete man als Hauptschauplätze die militärischen Wirren des Dreißigjährigen Krieges. Während der Gegenreformation erwies sich die Oberlausitz dann für die schlesischen Protestanten als Zufluchtsort. Und nicht zuletzt verbanden immer wieder herausragende Einzelpersönlichkeiten in ihrem Werk beide Landschaften: der Baumeister Wendel Roskopf nannte sich „Meister zu Görlitz und Schlesien“, die Mystik des Jakob Böhme wäre in ihrer Entstehung 
ohne Einflüsse aus Schlesien undenkbar, der Oberlausitzer Christoph Nathe wurde mit seiner Malerei zu einem der wichtigsten Entdecker des Riesengebirges. Eine Tradition, die sich nach 1815 fortsetzte: Richard Jecht beteiligte sich als Görlitzer Stadtchronist und Historiker der Oberlausitz auch am akademischen Leben in Breslau - und noch Arno Schmidt spielte in seinem Werk immer wieder mit den eigenen familiären und kulturellen Wurzeln in beiden Landschaften. All dies machte die Oberlausitz und Schlesien zu guten, engen Nachbarn. Dass aber das Land zwischen Riesengebirgskamm und Oderniederung aus Oberlausitzer Sicht einen besonderen Stellenwert eingenommen hätte, wie sich das in verschiedener Hinsicht für Böhmen behaupten ließe, dafür finden sich bis 1815 keine Überlieferungen.

\section{Gemeinsamkeiten und feine Unterschiede}

Zur Situation in Görlitz nach der Teilung der Oberlausitz infolge des Wiener Kongresses 1815 schrieb Max Kwiecinski 1902: „Die bezüglichen Erlasse wurden in tiefem Schmerz aufgenommen, der noch Jahre lang rege blieb. Während sich die älteren Leute niemals mit der preußischen Herrschaft befreunden konnten, wurde die Stimmung der jungen Generation allmälig eine andere, nachdem man eingesehen, was es für ein unschätzbarer Vortheil war, einem mächtigen Staate anzugehören, dessen Fürst bestrebt war, das Wohl seiner Unterthanen nach allen Richtungen zu fördern." In dieser Perspektive war es also ein langsames, zunächst auch widerwilliges Hineinwachsen in neue, zuvor nie da gewesene Gegebenheiten. Zwei Drittel der Oberlausitz wurden der Provinz Schlesien angegliedert. Spitz zu laufend wie ein Tortenstück ragte Schlesien, um die nordöstliche Oberlausitz erweitert, nun nach Westen. Dass hier eine Grenze am Kartentisch wie ein Schnitt durch eine über Jahrhunderte gewachsene Region gezogen worden war, ist klar ersichtlich. Kirchspiele und Gutsherrschaften wurden dabei genauso getrennt wie das sorbische Sprachgebiet. Und Görlitz fand sich plötzlich im Westen und Süden von einer Zollgrenze umgeben, hinter der sächsisches Ausland lag. Eine Situation, die noch dadurch weiter erschwert wurde, dass offenbar auch im „alten“ Schlesien die verinnerlichten Grenzen nicht so einfach aus den Köpfen zu bekommen waren: „Man redete mir in Glogau sehr ab, dorthin zu gehen; es bestand damals nur zweimal in der Woche eine Post- verbindung über Bunzlau, sonst aber nur ein äußerst geringer Verkehr. Man betrachtete es gewissermaßen noch als eine sächsische Stadt. Am 4. Januar 1831 abends spät traf ich in Görlitz ein und stieg im Hirsch, dem damals ersten Gasthof ab." So schildert Hugo Sattig, von 1857 bis 1866 Görlitzer Oberbürgermeister, in seinen Memoiren die Situation in der Neißestadt zu Beginn seiner hiesigen Karriere. Auch für den in Glogau Geborenen selbst ging fortan „nach Schlesien die Schnellpost wöchentlich nur zweimal."

Bis 1845 sollte sich diese Situation durch die Reformen in Preußen sowie das vorausschauende Handeln von lokalen Politikern wie dem ersten Görlitzer Oberbürgermeisters Gottlob Ludwig Demiani immer weiter verbessern. Ein Meilenstein für Görlitz und Umgebung war dabei der Anschluss an die SächsischSchlesische Eisenbahn. Am 7. August 1847 fuhr aus Sachsen von westlicher Seite her zum ersten Mal ein Zug in die Stadt ein. Dazu schrieb der Görlitzer Anzeiger: „Nach kurzem Verweilen begab sich die festlich geschmückte Lokomotive ,Lusatia' nach Reichenbach zurück." Nicht nur in Form der ersten je in Görlitz eingetroffenen Lokomotive blieb die Oberlausitz in der Stadt präsent. Man muss dazu nicht einmal das Wirken der Oberlausitzischen Gesellschaft der Wissenschaften hervorheben. Ob Verbrüderungsfest der Oberlausitz 1848, ob Oberlausitzer MännerGesangs-Fest auf der Landeskrone 1850, ob Turnfest auf der Landeskrone 1861 mit dem aus Görlitz stammenden „Turnvater der Oberlausitz" August Moritz Böttcher - die namentliche Bezugnahme auf die auf zwei deutsche Staaten verteilte Heimatregion riss in Görlitz nie ab.

Auch die Gründung des Deutschen Reiches änderte an dieser Situation nichts Grundlegendes. Und so hieß es bei Franz Schroller, als er 1885 in seiner „Schilderung des Schlesierlandes" auf den oberlausitzischen Landesteil und dessen wichtigste Stadt zu sprechen kam: „Görlitz gehört zwar der Verwaltung nach zu Schlesien; allein seine Geschichte, seine Lage und so manche Eigentümlichkeiten weisen es doch mehr auf Sachsen hin [...]. Man wird am richtigsten Görlitz und seine Umgegend als das Übergangsgebiet zwischen Sachsen und Schlesien bezeichnen können, denn dem reinen Sachsen wird hier wieder manches Schlesische auffallen. [...] Schlesiens Hauptstadt und das Schlesierland überhaupt sind verhältnismäßig nur wenigen Görlitzern bekannt; der Görlitzer Kaufmann hat naturgemäß mehr Beziehungen nach dem Westen und Norden, nach Leipzig und Berlin, als 
Oberlausitzer Ruhmeshalle in Görlitz, um 1900. Foto: Kulturhistorisches Museum Görlitz

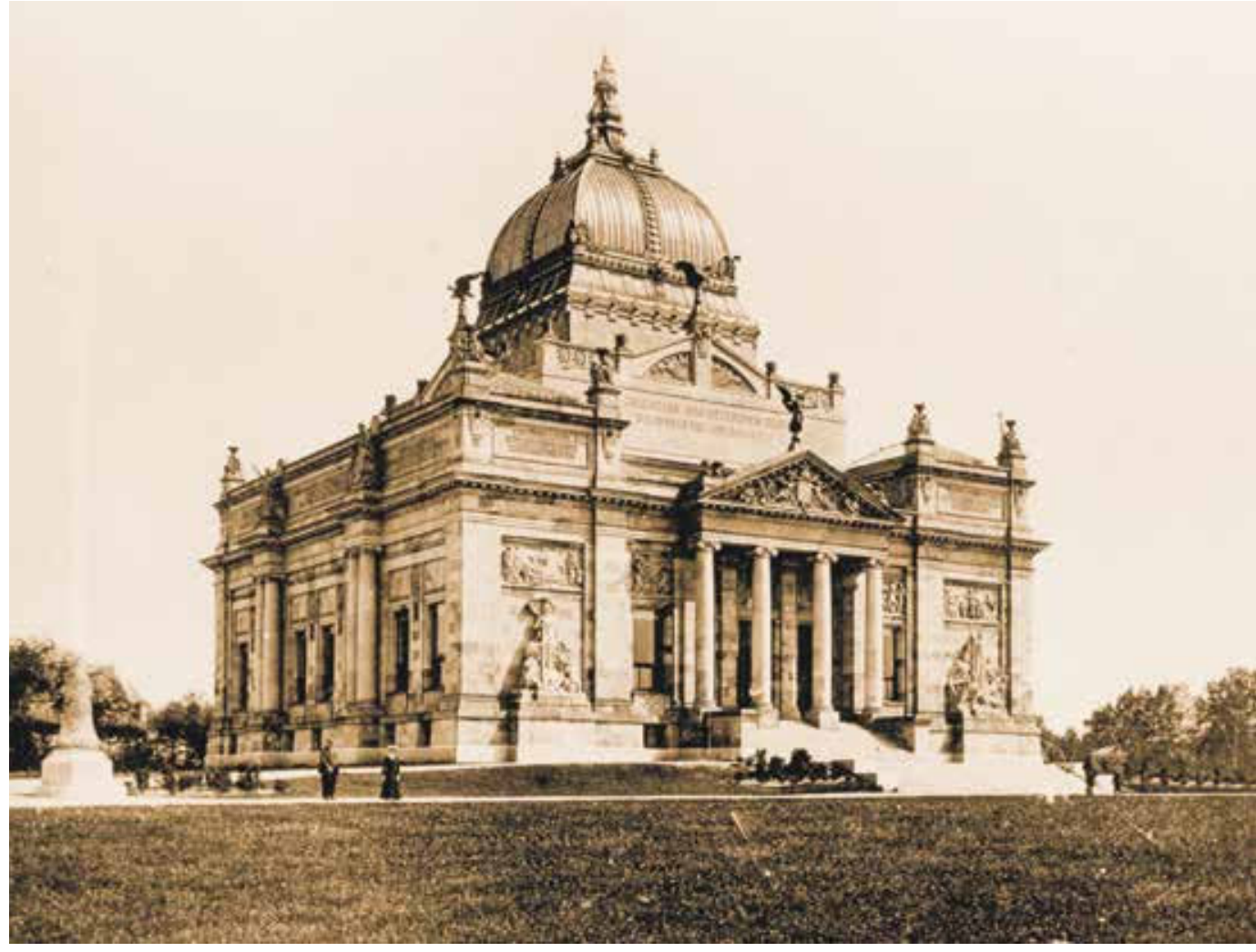

nach Breslau. “ Neben Görlitz erwähnte Schroller überhaupt nur noch Muskau: „Um dieses Parkes willen geschieht es, dass wir dieser, verlorenen Ecke' Schlesiens einen Besuch ab-statten." 1893 kam auch Kaiser Wilhelm II. wieder einmal „in diese Ecke“: zur Einweihung des von der Bürgerschaft eines im gründerzeitlichen Boom begriffenen Görlitz gestifteten Denkmals für Wilhelm I. auf dem Obermarkt, dessen nach Osten und damit in schlesische Richtung gewandte Seite das Oberlausitzer Wappen und die Inschrift „Dem Einiger Deutschlands die getreue und dankbare Oberlausitz 1893“ trug. In den folgenden Jahren folgten diesem Wappen im stetig wachsenden Görlitz weitere Oberlausitzer Bezugnahmen: die Altstadtbrücke trug das Wappen, der Rathausanbau die Wappen aller Sechsstädte, in der Südstadt erhielt ein ganzer Platz deren Namen, und noch in der Halle des neuen Bahnhofs von 1917 schmücken sie die Fenster. Den Höhepunkt dieser symbolischen Herausstreichung der Oberlausitz im Stadtraum stellte aber zweifellos die 1898 bis 1902 erbaute Oberlausitzer Ruhmeshalle auf dem östlichen Neißeufer dar. Durchweg aus Spenden finanziert, belegte das Bauwerk den wilhelminisch geprägten Patriotismus der Oberlausitz vor dem Ersten Weltkrieg. Die Ruhmeshalle war außen wie innen förmlich übersät mit Bezugnahmen zur regionalen Geschichte. Da waren die in allen vier Himmelsrichtungen am First angebrachten Widmungssprüche von herausragenden Mitgliedern des Bauausschusses. Besonders markant war der auf der Nordseite, von der aus man sich dem Bau aus Richtung Innenstadt kommend annäherte. Er stammte vom Initiator des Projektes, dem Görlitzer Bürgermeister Johannes Heyne und lautete: „Gott zum Dank, unserem ruhmreichen Hohenzollernhause zur Ehre, der Kunst zur Weihe und Pflegestätte, der Oberlausitz zur Stärkung der Heimatsliebe." Durch die Eingangstüren mit den Wappen der Sechsstädte ging es in die Haupthalle, vorbei am Wappen der Oberlausitz und am Görlitzer Stadtwappen im Eingangsbereich. Rund um diese Halle erstreckten sich die Räumlichkeiten des Kaiser-Friedrich-Museums, das sich als „Provinzialmuseum der Preußischen Oberlausitz" ganz der Vermittlung Oberlausitzer Geschichte verschrieben hatte und dessen Rundgang den „Oberlausitzer Ehrensaal“ einschloss. Noch in seinem Führer durch die Ausstellung von 1910 erwähnt der Museumsdirektor Ludwig Feyerabend Schlesien mit keinem Wort. Und bereits 1903 hatte Bürgermeister Heyne auf die Anfrage aus Breslau nach eventuellen Görlitzer Beiträgen für ein dortiges Sammlungsprojekt unmissverständlich ablehnend geantwortet: „Wir haben in 
Görlitz unser eigenes Museum und sammeln Oberlausitz. Wir werden auch möglichst wenig nach Breslau geben von der Oberlausitz."

Das klingt fast einhundert Jahre nach dem Wiener Kongress nach sehr stark betonter Eigenständigkeit und Andersartigkeit. Sollte sich die lange Zeit der gemeinsamen Verwaltung überhaupt nicht in Görlitz niedergeschlagen haben? Schlesien - in Görlitz ein Tabubegriff? Eine solche Behauptung läge fern der Realität. Bereits seit 1863 erschien in Görlitz die weit nach Niederschlesien hinein vertriebene „Niederschlesische Zeitung“. 1885 hatte die Görlitzer Sektion des Riesengebirgsvereins auf der Industrie- und Gewerbeausstellung ihren großen Auftritt: der von ihr errichtete Nachbau der Teichbaude wurde zu einem Magneten der Ausstellung. Überhaupt war Görlitz in diesen Jahren ein Einfallstor für die preußische Riesengebirgsbegeisterung aus Richtung Berlin. Görlitzer Abiturienten gingen selbstverständlich auch nach Breslau zum Studium, auch wenn sie sich dort womöglich dem Corps Lusatia, einer angesehenen Studentenverbindung, anschlossen. Görlitzer Wehrpflichtige verrichteten ihre Dienstzeit in schlesischen Garnisonsstützpunkten wie Glogau oder Liegnitz. Auch das schlesische Wappen war auf der Altstadtbrücke vertreten; im Rathausanbau zierte es immerhin eines der farbigen Schmuckfenster im Treppenhaus. Und nicht nur für die zahlreich zugezogenen Pensionäre wurde seit 1878 das Schlesische Musikfest zum regelmäßigen Höhepunkt des gesellschaftlichen Lebens der Neißestadt. Görlitz war in der Zeit der Jahrhundertwende zur zweitgrößten Stadt Niederschlesiens nach Breslau geworden - und das zeigt man stolz bei jeder sich bietenden Gelegenheit. So im Jahr 1905, als man rund um die frisch errichtete Ruhmeshalle die Niederschlesische Gewerbe- und Industrieausstellung abhielt. Den über 1,5 Millionen Besuchern präsentiert sich die Stadt hier ganz selbstbewusst als Hauptstadt der Oberlausitz innerhalb Schlesiens. Auf dem linken Eingangsturm zum Ausstellungsgelände wehte die Fahne der Oberlausitz, auf dem rechten die Fahne der Stadt Görlitz, im Hintergrund feierte der Spruch von Bürgermeister Heyne die Heimatliebe der Oberlausitzer - und zwischen all dem präsentiert sich die Industrie Schlesiens. Man hatte es in Görlitz gelernt, die Heimatregion innerhalb Schlesiens als eigenen Repräsentationsbereich zu markieren, in den "die richtigen Schlesier" nicht hinein zu reden haben. Das hatte mit übergroßem Zusammengehörigkeitsgefühl zu den einstigen Schwesterstädten des Sechsstädtebundes vermutlich recht wenig $\mathrm{zu}$ tun, denn die hatte man als „kleine Großstadt“ zwischen Dresden und Breslau längst weit hinter sich gelassen. Schon 1861 klang das in den Worten des Oberbürgermeisters Hugo Sattig an, als er in Breslau zu seinem königlichen Landesherren sprach: „Majestät, Görlitz ist, seit es unter dem Zepter der Hohenzollern steht, so reich begnadigt und so zu einer Blüthe entfaltet worden, dass wir dafür nur im höchsten Grade dankbar sein können. Seine Nachbarstadt Bautzen war zur sächsischen Zeit die bedeutendste Stadt der Oberlausitz. Seit Görlitz aber preußisch ist, hat es Bautzen weit überholt, und das haben wir dem preußischen Regiment zu verdanken."

Görlitz war vor dem Ersten Weltkrieg der auf Sachsen und Preußen aufgeteilten Oberlausitz längst entwachsen. Kulturpolitisch nutzt man die besondere Geschichte der Region zur Herausstreichung der eigenen Selbstständigkeit innerhalb Schlesiens. Im Alltag der Stadt jedoch führten im Görlitzer Adressbuch von 1913 der Gartenbauverein für die Oberlausitz und der Schlesische Farben-Kaninchen-Züchter-Club ein friedvolles Nebeneinander. Und am Blockhaus über dem Neißeviadukt versammelten sich bei guter Fernsicht Einheimische wie Touristen und genossen die Aussicht zur Schneekoppe.

\section{„Starkes Bollwerk des Deutschtums im Osten“}

Nach dem Ersten Weltkrieg geriet die Stadt in wirtschaftlich schwereres Fahrwasser. Der Börsencrash machte viele der vormals wohlhabenden Pensionäre zu Sozialfällen. Und auch bei den Arbeitslosenzahlen rangierte man hinter Breslau jahrelang auf dem zweiten Platz. Der Görlitzer Arbeiterschriftsteller Albert Klaus hat mit dem in der Stadt angesiedelten Roman „Die Hungernden“ dieser Situation 1932 ein bedrückendes literarisches Denkmal gesetzt. Die in Not geratene Arbeiterschaft las zu diesem Zeitpunkt nicht mehr, wie noch 1902, in der „Görlitzer Volkszeitung - Organ für die werktätige Bevölkerung der Ober-Lausitz“. Vielmehr trug das Blatt der Görlitzer SPD in diesem Jahr bereits den Zusatztitel „Organ für die werktätige Bevölkerung Niederschlesiens“. Dieser Wechsel in der regionalen Zuordnung dürfte für die notleidende Arbeiterschaft in Görlitz zweitrangig gewesen sein.

Von größter emotionaler Wichtigkeit waren hingegen deutschlandweit die als Fanal empfundene Teilung Oberschlesiens und die zunehmende nationalistische Spannung zwischen Deutschen, Polen und Tschechen in der 
Region. Bereits 1902 hatte Max Kwiecinski zur Einweihung der Bismarcksäule auf der Landeskrone eine entsprechende Tonlage angestimmt: „Von diesem Platze aus wird auch das Feuer hinüberleuchten zu unsern mit deutscher Kraft um das Deutschthum ringenden Brüdern jenseits der österreichischen Grenze“. 1927 las sich das beim Hirschfelder Reichstagsabgeordneten Max Schmidt unter dem Titel „Die preußische Oberlausitz als Grenzgebiet“ dann bereits so: „Zu den den Frieden gefährdenden Unruheherden gehören an den Grenzen Schlesiens zweifellos die Polen und Tschechen. Letztere führen [...] mit allen ihnen zu Gebote stehenden staatlichen und privaten Mitteln einen bewussten Feldzug in politischer, wirtschaftlicher und sozialer Hinsicht gegen alles Deutsche [...]. Gegenüber diesem Treiben und diesen Bestrebungen ist es notwendig, dass die preußische Oberlausitz und ihr Umland bei kräftiger Gegenwehr durch Selbsthilfe auch der besonderen staatlichen Unterstützung und Fürsorge bedarf." Diese äußerst angespannte Tonlage durchzog den gesamten 1927 herausgebrachten Präsentationsband der „Preußischen Oberlausitz“. Sei es der Görlitzer Oberbürgermeister Snay: „Zum Schluß muß noch darauf hingewiesen werden, welche außerordentliche Bedeutung die Stadt Görlitz für das Deutschtum als Grenzstadt hat." Sei es im Beitrag zur Landwirtschaft der Region: „Denn das Schlesien und mit ihm die Oberlausitz nach zwei Seiten Grenzland ist und damit besonders hohe Aufgaben für das Deutschtum zu erfüllen hat, ist leider im übrigen Deutschland zu wenig erkannt." Sei es im Beitrag zum Sportwesen der Gegend: „Die Oberlausitz ist ein altes Kampfland, das einst als deutsche Grenzmark, später als kampfdurchtobter Provinzteil in ewigen Sorgen und Nöten war." Oder sei es bei Ludwig Feyerabend, der über die Ruhmeshalle und das Museum schrieb: „Begeisterte Liebe zur Oberlausitzer Heimat, vereint mit hochherziger Opferwilligkeit für ihre idealen Güter schuf einen Inhalt, der heute [...] ein Bollwerk deutscher, Oberlausitzer Kultur im deutschen Grenzlande bildet [...].“

Dass in keinem der genannten Beiträge ein Hinweis darauf fehlt, wie dringend die öffentlichen Kassen dieses „deutschen Grenzlandes“ auf Zuwendungen aus dem Reich angewiesen wären, um den "jahrhundertealten Kampf “ gegen die bedrohlichen Nachbarn aufrecht erhalten zu können, zeigt zum einen, dass auch in der nationalistisch extrem aufgeheizten Weimarer Republik die Kommunalpolitik im sprachlichen Gestus der Zeit „ihren Stich“ zu machen suchte. Es zeigt aber auch, dass auf diesem Weg bereits ein Stück des noch die Zeit der Jahrhundertwende bestimmenden Görlitzer Spiels mit den Identitäten zwischen Oberlausitz und Schlesien verloren gegangen war. Zum ersten Mal überhaupt findet sich in dem Band von 1927 eine Karte, die mit „Schlesischer Oberlausitz" unterschrieben ist. Auch wenn es in den verschiedenen Texten dann stets noch "Preußische Oberlausitz" heißt - ein Anfang war damit gemacht. Die alten Bande begannen lockerer zu werden, die feinen historischen Unterschiede wichen den „großen Erzählungen“ des 20. Jahrhunderts.

Noch im Bewusstsein dieser Unterschiede spöttelte Johannes Wüsten in den 1930er Jahren über seine Heimatstadt: „In der Oberlausitz ist der schlesische Einschlag schon recht spürbar und der Dialekt entsprechend hanebüchen. [...] Mit Görlitz selbst haben die verschiedenen Friedenskongresse die verschiedensten Blödheiten angestellt, es hat so ziemlich allen umliegenden Regentschaften angehört, hauptsächlich Böhmen und Sachsen. Seit 1814 [sic!] gehört die Stadt zu Schlesien, zu dem sie bestimmt nicht gehört. Schlesien ist ganz was anderes als die Oberlausitz. Breslau zum Beispiel ist schlesisch. Görlitz unter gar keinen Umständen! Aber die Regierung hat das noch nicht gemerkt, und die Oberlausitzer haben noch keine eigene Partei gegründet, was übrigens den Krieg gegen Schlesien bedeuten würde. Einstweilen sind wir hier so ein Übergang, und wer die sächsische und die schlesische Sprache beherrscht, tut wohl daran, er kann sich so vollauf verständigen."

Die Oberlausitzer sind dann doch nicht gegen die Schlesier in den Krieg gezogen, sondern vereint mit ihnen unter der Hakenkreuzfahne gegen die Nachbarn. Johannes Wüsten, der als Görlitzer Künstler selbstverständlich auch Rübezahl zu seinen Sujets zählte, überlebte als Kommunist die NS-Gefängnishaft nicht. Und Arno Schmidt siedelte den Untergang seiner Jugend, ihrer Welt und der sie tragenden Gesellschaft im "Leviathan“ in einem Eisenbahnwagon an, hoch auf dem zerstörten Görlitzer Viadukt über der Neiße, im Nirgendwo zwischen Ost und West, den Weg zurück nach Schlesien selbst zerstört mit einem „Schienenwolf“, den der Zug auf der Flucht hinter sich her gezogen hatte. Eine ganze Welt war unwiederbringlich im nationalsozialistischen Furor untergegangen.

\section{Verschwundene Landschaften?}

Hatte das Jahr 1815 bereits einen nachhaltigen Einschnitt in das Gefüge der Region bedeutet, so sollte dies für das Jahr 1945 um ein Vielfa- 
ches potenziert gelten. Mit der Auflösung des Landes Preußen nach der deutschen Kapitulation geriet zwar die geteilte Oberlausitz wieder unter eine einheitliche sächsische Landesverwaltung. Aber bereits 1952 wurde dieser Zustand mit der Abschaffung der Länder in der DDR und der Gründung der Bezirke Dresden und Cottbus beendet. Der nördliche Streifen der Region mit Ruhland, Hoyerswerda, Weißwasser und Bad Muskau gehörte nun zum „Energiebezirk der DDR“, und dessen alleinige Ausrichtung auf die Braunkohleindustrie sollte diesen Teil der Oberlausitz entscheidend verändern. Noch einschneidender war die Situation aber entlang der Neiße. Über Nacht war der Fluss zum ersten Mal in seiner Geschichte überhaupt zum Grenzfluss geworden, an dem entlang nun zusätzlich noch eine neue Sprachgrenze verlief. Das Gebiet östlich von Zittau, die östlichen Teile der Kreise Rothenburg und Görlitz und der ganze Kreis Lauban standen nun unter polnischer Verwaltung und gehörten kurz darauf zur Volksrepublik Polen. Der Görlitzer Kommerzienrat Conrad Heese prophezeite in seinem Tagebuch am 1. August 1945: „Wenn Görlitz-Ost polnisch wird, ist unser Vermögensverlust ein totaler und Görlitz überhaupt ruiniert. Sein Reichtum, - die Görlitzer Heide - Gas- und Elektrizitätswerke der beste Teil der Landwirtschaft des Kreises -, liegt östlich der Neiße."

Diese Analyse war wirtschaftspolitisch von einiger Voraussicht. Es kostete die DDR große Anstrengungen, die Wirtschaftskraft der geteilten Stadt wieder auf die - nun planwirtschaftlichen - Füße zu stellen. Das geteilte Görlitz hatte aber in den ersten Nachkriegsjahren ein viel sichtbareres Problem als das zerstörte wirtschaftliche Gefüge: Wie Markus Lammert in seiner Studie über Görlitz nach 1945 herausstellte, war die Grenzstadt mit Flüchtlingen und Vertriebenen „von drüben“, wie es nun bald hieß, hoffnungslos überfüllt. Am Ende der 1940er Jahre waren etwa 40 Prozent der Einwohner Flüchtlinge und Vertriebene, ganz überwiegend aus Schlesien. Das war der Spitzenwert in der DDR. In der deutschen Stadthälfte leben zeitweise 120.000 Menschen, womit sie die am dichtesten bevölkerte Stadt der sowjetischen Zone darstellte. 1945 war die Lage in Görlitz schlicht katastrophal. In den Monaten nach Kriegsende beklagte man über 1000 Hungertyphus-Tote, unter ihnen im August auch der Görlitzer Oberbürgermeister. Die Säuglingssterblichkeit lag bei 90 Prozent, die Stadt war nicht mehr in der Lage, die sich in ihr aufhaltenden Menschen ausreichend mit Wohnraum und Nahrung zu versorgen. Noch

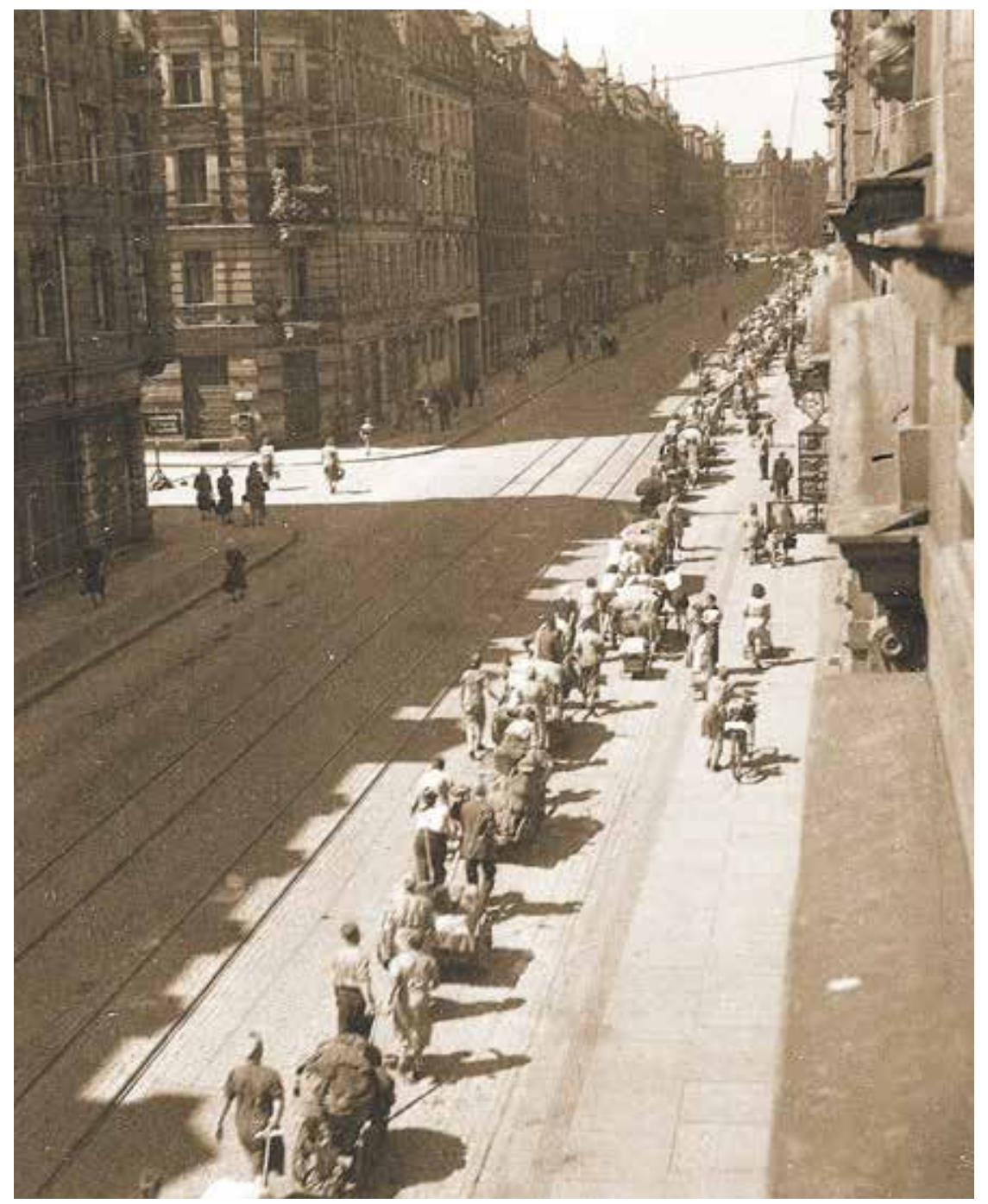

1948 bezeichnete ein Zeitungsartikel Görlitz als "Armenhaus an der Grenze“. Bezogen auf das erste Jahrzehnt der DDR, spricht Markus Lammert von Görlitz als „Stadt der Vertriebenen" und „schlesischer Exilstadt".

Diese Zuschreibung ist keineswegs eine Neuprägung der Gegenwart. Aus den unmittelbaren Nachkriegsjahren finden sich im Görlitzer Ratsarchiv mehrere Akten, die belegen, dass zumindest ein Teil der Görlitzer Bevölkerung die Stadt in dieser Weise wahrnahm. So entspannten sich unmittelbar nach dem Tod Gerhard Hauptmanns im Sommer 1946 unterschiedliche Bemühungen, den Nachlass des Dichters dauerhaft nach Görlitz zu bekommen. In einer Eingabe des Lehrerbildungskursus liest sich das am 13. Juni 1946 so: „Unsere Anstalt für Neulehrerkurse trägt daher Ihnen, sehr geehrter Herr Oberbürgermeister, die Bitte vor, dass Görlitz als die letzte verbliebene schlesische Stadt alle Kräfte einsetzt und alle Möglichkeiten ausnutzt, um von dem Hab und Gut Hauptmanns soviel wie möglich sicherzustellen.
Flüchtlinge aus Schlesien in Görlitz, 1945.

Foto: Ratsarchiv Görlitz 


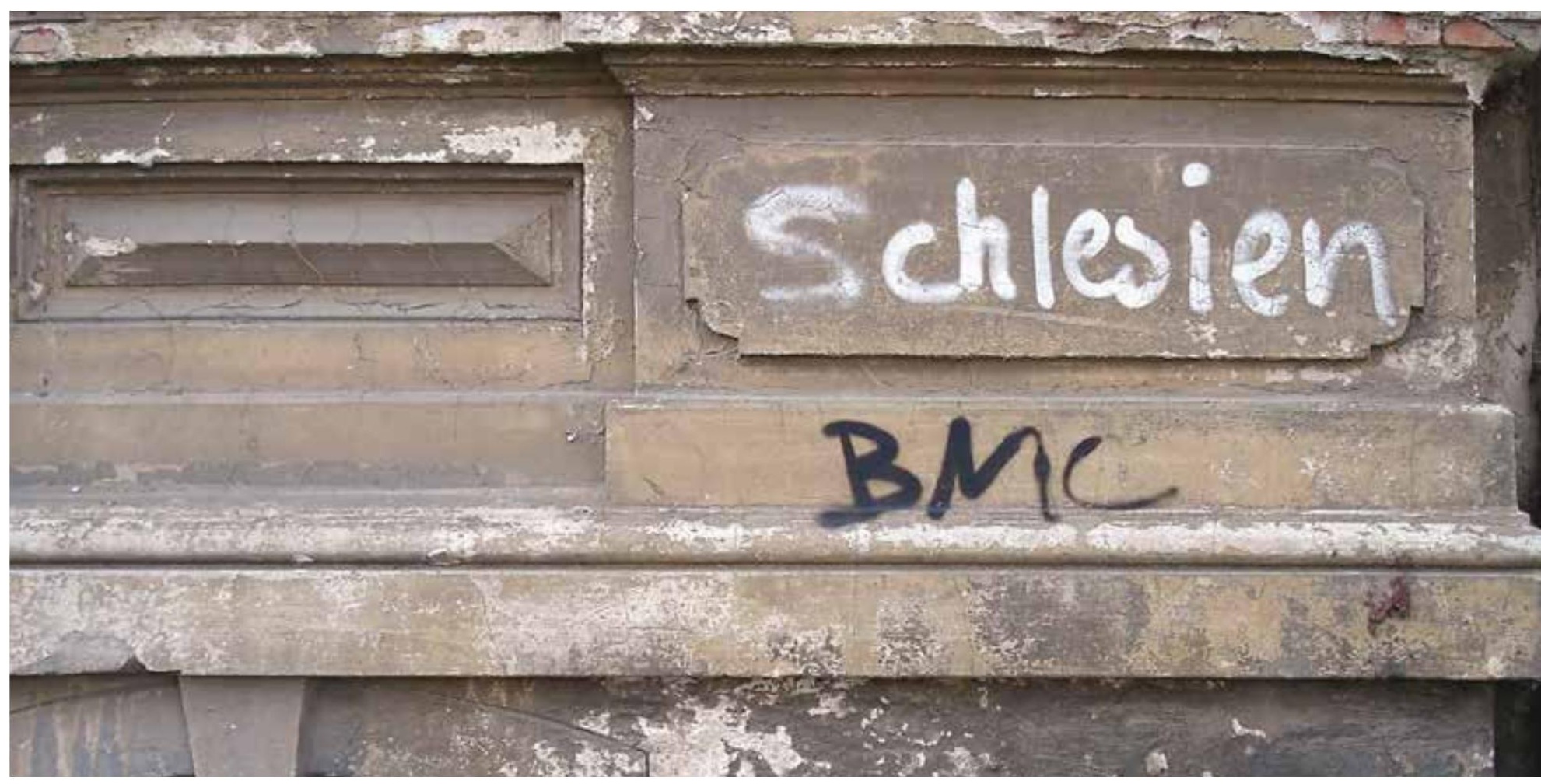

Wandaufschrift beim Südausgang des Görlitzer Bahnhofs, 2008. Foto: Robert Lorenz
Gerhard Hauptmann hat sich in den Mauern unserer Stadt immer wohlgefühlt." Zur Bekräftigung nannte man in diesem Sommer auch das Görlitzer Stadttheater in "Gerhard-Hauptmann-Theater" um. Und in der Ratssitzung vom 31. Juli 1946 beschloss der Stadtrat die Einrichtung eines Schlesischen Museums im Görlitzer Kaisertrutz.

Führt man sich vor Augen, wie wichtig nur wenige Jahrzehnte zuvor den Museumsverantwortlichen in Görlitz noch die Abgrenzung zu Schlesien gewesen war, wird deutlich, wie einschneidend der Bruch von 1945 empfunden worden sein muss. Angesichts der völligen Auflösung der alten Nachbarschaften und der mit ihnen zusammenhängenden Alltags- und Namenswelt, angesichts des alltäglichen Elends der unfreiwillig hinzugekommenen neuen Mitbürger, war den noch bei Johannes Wüsten anzutreffenden und mit Humor ausgetragenen "regionalistischen Hakeleien" zwischen Oberlausitz und Schlesien, wie man sie in Görlitz 130 Jahre lang gepflegt und zur Interessenwahrung auch stadtpolitisch kultiviert hatte, quasi über Nacht die Grundlage entzogen worden. Womöglich war Görlitz in der Wahrnehmung vieler seiner Bewohner nie "schlesischer" als zum Zeitpunkt des empfundenen Untergangs von Schlesien. Einige nahmen dieses Gefühl wohl weiter mit nach West-Deutschland. So bemerkte der Pfarrer und Kirchenhistoriker Christian-Erdmann Schott: „Görlitz war ein Symbol dafür, dass Schlesien nicht völlig unter- gegangen war, dafür, dass Schlesier irgendwo doch noch ein kleines Stück Heimat besaßen. Und dieses Stück Heimat hieß: Görlitz." Doch auch in der östlichen Oberlausitz sollte mit den sich hier von Breslau her ,in dem sogenannten Restschlesien", wie Bischof Ernst Hornig in Görlitz im Oktober 1948 schrieb, ansiedelnden evangelischen und katholischen Kirchenleitungen Schlesiens eine lokalspezifische Erinnerungstradition als Flüchtlingskirchen begründet werden, die sich die DDR-Jahre hindurch unter ihren Bischöfen, Pfarrern und vielen Gemeindegliedern erhielt.

Allerdings musste diese Erinnerungsleistung im Gegensatz zur westdeutschen Situation bald inoffiziell erfolgen. Denn im Jahr 1950 vollzog die DDR in einem feierlichen Akt die Anerkennung der neuen Grenze zur Volksrepublik Polen - im einstigen Ost-Görlitz, das nun Zgorzelec hieß und in dem man für diesen Anlass die vormalige "Ruhmeshalle" als passenden Ort bestimmt hatte, die nun das „Dom Kultury" der neuen polnischen Bewohner war. Damit galt das Thema für abgeschlossen und eine zu stark nach außen getragene Beschäftigung mit der schlesischen Herkunft in der DDR als unerwünscht und verdächtig. In einer Stadt voller ehemaliger Schlesier musste so eine Geschichtspolitik auf Widerstände stoßen. So berichtete bereits eine Aktennotiz des Görlitzer Amtes für Volksbildung vom 28. Februar 1949 in allarmierendem Tonfall vom „Gastspiel des Mundartsprechers Paul Heinke in Görlitz“. 
Dieses „trug eine stark schlesische, wenn nicht schon gar chauvinistische Tendenz. Die Umrahmungen Paul Heinkes zu seinen Vorträgen waren dazu angetan, in den Neubürgern ein starkes Sehnsuchtsgefühl nach ihrer alten Heimat zu wecken. [...] Herr Heinke hat die Genehmigung, „Lausitzer Heimatabende“ durchzuführen, was jedoch nicht geschehen ist. Aus dem Verwandtschaftsverhältnis der lausitzer und der schlesischen Mundart war es Herrn Heinke sehr leicht möglich, in Görlitz einen ausgesprochen schlesischen Heimatabend $z u$ veranstalten, indem die im Manuskript angegebenen lausitzer Gedichte statt der Bezeichnung "Lausitz“ oder „Oberlausitz" stets mit den Worten „Schlesien“ oder „Oberschlesien“ vorgetragen wurden." Die „Neubürger“ nutzten zunehmend auch beliebte Ausflugspunkte mit Fernblick in Richtung Riesengebirge wie die Landeskrone und den Kreuzberg bei Jauernick für ihren Wochenendspaziergang mit der Familie. Ein besonders emotionaler Ort sollte aber der „BlockhausBlick" am zerstörten Eisenbahnviadukt werden. Der letzte katholische Pfarrer der einstigen Oststadt, Franz Scholz, hatte bereits 1946 unmittelbar nach seiner Ausweisung die Gefühlslage der nächsten Jahre für viele Besucher an diesem Ort beschrieben. Am 17. Juni 1946 notierte er in seinem Tagebuch: „Vom Blockhaus in Görlitz-West, am gesprengten Viadukt, schaue ich jetzt über die Neiße in das schlesische Land. Nur einen Kilometer entfernt liegt meine Kirche St. Bonifatius, die Glocken läuten wie immer."

Natürlich veränderte sich in vierzig Jahren DDR diese Gefühlswelt allmählich. In den 1960er Jahren wurde die Grenze nach Polen durchlässiger. Viele unternahmen mit der Familie Ausflüge an den alten Wohnort jenseits des Flusses. Häufig ergaben sich Begegnungen mit den neuen Bewohnern der alten Häuser, aus denen sich in einigen Fällen auch Freundschaften entwickelten. Im Winter fuhr man wieder zum Skilaufen ins Riesengebirge. In den 1970er Jahren befanden sich Görlitz und Zgorzelec das erste Mal für kurze Zeit auf dem Weg zur Doppelstadt. Es gab erste Eheschließungen über den Fluss hinweg, und man machte sich Gedanken zu einer gemeinsamen Stadtplanung. Im Presseladen des „Dom Kultury“ kauften sich die Görlitzer westdeutsche Zeitungen. Nach außen hin waren die „Neubürger" nur noch am Dialekt von den übrigen Bewohnern in der Stadt und der Region zu unterscheiden. Sie hatten in Görlitz den industriellen Wiederaufbau mit geleistet und die Stadt hinter Dresden zur Nummer Zwei im Bezirk gemacht, waren Kollegen „in der Kohle“ und in der LPG. Auf den Dörfern waren sie Teil des dortigen Heiratsmarktes geworden, was in den Heidedörfern für den Alltagsgebrauch der sorbischen Sprache zu einer zusätzlichen Herausforderung werden sollte, die nur in wenigen Fällen gemeistert wurde.

Eine gemeinsame Identitätskonstruktion erfolgte öffentlich im Sinne der propagierten sozialistischen Gesellschaft über die Betriebe und in den Strukturen der Kreise und Bezirke. Schlesien spielte dabei selbstverständlich entlang der Neiße keine Rolle mehr. Die Oberlausitz allerdings auch nicht. Die alten Regionalismen waren absichtsvoll durch neue ersetzt worden. Privat und im Kreis von Geschichtsfreunden konnte man sich durchaus mit der regionalen Historie vor 1945 beschäftigen. Doch eine Identitätserzählung in Anlehnung an den Sechsstädtebund oder gar das ständisch orientierte Markgraftum Oberlausitz waren weder im Heimatkundeunterricht an den Schulen ein Thema, noch wurden sie in der „Stadt der Türme“, wie Görlitz für sich in der DDR warb, hervorgehoben. Hinzu kam, dass die wissenschaftliche Beschäftigung mit der Regionalgeschichte nach der Auflösung der Oberlausitzischen Gesellschaft in Görlitz 1945 ihren Schwerpunkt an das 1951 gegründete Sorbische Institut in Bautzen verlagert hatte. Hier stand nachvollziehbarer Weise das sorbische Siedlungsgebiet innerhalb der beiden Lausitzen im Vordergrund der Arbeit und damit eine räumlich wie geschichtlich anders gewichtete Sicht auf die Region, die in den östlichen Teil der Oberlausitz weniger Verbindungen hatte. So verband sich mit dem Begriff "Lausitz" nun republikweit eine sorbisch geprägte Vorstellung der Gegend. Und mit der "Oberlausitz" die FDGB-Ferienregionen des Oberlausitzer Berglandes und des Zittauer Gebirges. Görlitz und sein Umland spielten in dieser Wahrnehmung keine herausragende Rolle mehr.

Allerdings blieben in der viel beschriebenen DDR-„Nischengesellschaft“ die Erinnerungen an die solcherart scheinbar verschwundenen Landschaften im Privaten vielfach existent. Ines Geipel beschreibt es in einem Nachwort zu einem Roman der im Nachkriegs-Görlitz aufgewachsenen Sylvia Kabus so: „Die alten Städte der Lausitz. Ihre einzigartige Assimilationswelt. Das Gemisch aus verschiedenen Sprachen, Kulturen, Konfessionen, politischen Machtansprüchen. Sorben und Schlesier, Polen und Deutsche. Die Oder und das Riesengebirge, Provinz und Grenzland. [...] Eine Nachkriegskindheit aus Fülle und Raum also, aus Pracht und Tiefe. Doch in den Sog des Schönen einge- 
lassen harsche historische Konturen. Denn die Stadt, zwischen 1815 und 1945 zur preußischen Provinz Schlesien gehörend, wurde nach Kriegsende in das deutsche Görlitz und das polnische Zgorzelec geteilt. Von da an trennte der Grenzfluss Neiße Menschen, Identitäten, Erinnerungen, Ressentiments. Sylvia Kabus nennt ihre Kindheit denn auch einen "einzigen Ausnahmezustand, rauschauslösend, sinnenreich, bitter", in der Schlesien, Preußen und eben Vertreibung zu Unworten wurden und sich die eigene, innere Welt früh schon auf Verborgenes, Abwesendes, Verschwundenes ausrichtete."

Seit dem Herbst 1989 ist es mit dieser oft als traumatisch erinnerten Heimlichkeit im Umgang mit Schlesien in der Oberlausitz vorbei. In den letzten zwanzig Jahren haben sich hier sehr unterschiedliche Formen herausgebildet, das Zugehörigkeitsgefühl zu Schlesien zu leben und nach außen hin zu zeigen. Vom engen regionalen Horizont über den zum Teil auch aggressiv behaupteten, deutsch-nationalen Blick bis zum postnationalen, europäischhybriden Schlesienentwurf ist alles vertreten. Es muss sich noch zeigen, welche Kraft diese Rückbesinnung entwickeln kann und ob sich aus ihr heraus womöglich ganz neue schlesisch-oberlausitzische Erzählungen ergeben, die wir uns jetzt noch gar nicht vorstellen können und die in eine mögliche Zukunft der Region weisen, anstatt sich wie bisher hauptsächlich ihrer Vergangenheit zu vergewissern. Es wäre nicht verwunderlich, wenn sich dies einmal mehr in der Doppelstadt Görlitz-Zgorzelec vollzöge. Einstweilen fährt als ein möglicher Beginn seit 2012 hier wieder ein Zug auf Arno Schmidts Schulweg über Lauban (Luban) ins Riesengebirge.

\section{Literatur}

- Ines Anders: Die Oberlausitzer Gedenkhalle mit Kaiser-Friedrich-Museum in Görlitz 1902 bis 1932. Ein Beitrag zu Geschichte und Selbstverständnis der Städtischen Kunstsammlungen Görlitz. Berlin 1992.

- Ludwig Feyerabend: Führer durch die Gedenkhalle mit Kaiser-Friedrich-Museum. Görlitz 1910.

- Ines Geipel: In der Stummheit der Häuser. Nachwort. In: Sylvia Kabus: Weißer als Schnee. Frankfurt am Main 2008.

- Ingeburg Heese (Hrsg.): Aus dem Tagebuch von Justizrat Conrad Heese, Görlitz 1945. Oldenburg, Görlitz 2002.

Zum Weiterlesen: Lars-Arne Dannenberg, Matthias Donath, Dietrich Scholze (Hrsg.): Oberlausitzer Mythen. Meißen 2012, 206 Seiten mit zahlreichen Abbildungen, 17,00€.

Zu beziehen bei: Redaktions- und Verlagsgesellschaft Elbland $\mathrm{GmbH}$ Niederauer Straße 43 01662 Meißen Tel.: 03521-41045520 E-Mail: sz.meissen@dd-v.de

Es handelt sich um die überarbeitete Fassung des Artikels "Schlesien" in: Dannenberg, Donath, Scholze (Hrsg.): Oberlausitzer Mythen. Meißen 2012. Seite 190-201.
- Arno Schmidt: Leviathan oder Die Beste der Welten. Hamburg, Stuttgart 1949.

- Franz Scholz: Görlitzer Tagebuch. Chronik einer Vertreibung 1945/46. Frankfurt am Main, Berlin 1993.

- Christian-Erdmann Schott: Spuren und Wirkungen der schlesischen evangelischen Kirche im Nachkriegsdeutschland. Würzburg 2000.

- Franz Schroller: Schlesien. Eine Schilderung des Schlesierlandes. Glogau 1885.

- Erwin Stein (Hrsg.): Die Preußische Oberlausitz. Berlin 1927.

- Johannes Wüsten: Die Verrätergasse. Stücke, Aufsätze, Gedichte, Autobiographisches, Briefe. Berlin 1980.

Autor

Robert Lorenz

Berlin 